establish the most effective means of delivering this therapy.

Ruth Kirby

Original article Ambrosini A et al. (2005) Suboccipital injection with a mixture of rapid- and long-acting steroids in cluster headache: a double-blind placebo-controlled study. Pain 118: 92-96

\section{A new method for assessing stroke in the emergency room}

In view of the fact that many patients with stroke present to the emergency room, recognition of the signs and symptoms of stroke by emergencyroom physicians is crucial if early intervention is to be achieved. A UK group has developed a new stroke recognition tool for use in this setting, which promises to improve the differentiation of acute stroke from other conditions.

The design of the instrument-termed the Recognition of Stroke in the Emergency Room (ROSIER) scale-began with a 1-year developmental phase, involving 343 adult referrals from the emergency room to the acute stroke team. By recording the clinical characteristics of the patients with confirmed stroke or transient ischemic attack $(n=176)$ and those without stroke $(n=167)$, the researchers developed a seven-item stroke recognition tool. This tool covered loss of consciousness or syncope, seizures, asymmetric weakness of the face, arm or leg, and problems with speech or the visual field. Using a simple proforma to generate a 'ROSIER score', the instrument could then be used to determine the likelihood of stroke.

Following an internal validation process, the team carried out a 9-month prospective validation of the instrument. During this period, the proportion of non-stroke cases referred from the emergency room fell significantly. The ROSIER instrument "has good diagnostic accuracy and is simple to administer," say the researchers, and might be useful in the early recognition of stroke by emergency-room staff.

Ruth Kirby

Original article Nor AM et al. (2005) The recognition of stroke in the emergency room (ROSIER) scale: development and validation of a stroke recognition instrument. Lancet Neurol 4: 727-734

\section{Mid-life cardiovascular risk factors and dementia}

Two recent studies indicate that the development of dementia and Alzheimer's disease (AD) is affected by cardiovascular risk factors in mid life.

Researchers from Scandinavia reexamined 1,449 individuals aged $65-79$ years from the Cardiovascular Risk Factors, Aging, and Incidence of Dementia (CAIDE) study group, who had previously been recruited at mid life.

In the first study, the relationship between mid-life BMI and development of dementia or $A D$ was investigated, as the association between clustering of vascular risk factors and disease development. Mid-life obesity was associated with an increased risk of dementia and AD. High total cholesterol, high systolic blood pressure and mid-life obesity were all significant risk factors for dementia, increasing the risk of its development approximately twofold. Combination of these risk factors had an additive effect, such that the odds ratio for individuals with all three risk factors was $6.21(95 \% \mathrm{Cl}$ 1.94-19.92).

In a separate study, researchers looked at the association between leisure-time physical activity in mid life, and development of dementia or AD. Mid-life leisure-time physical activiy at least twice a week was associated with approximately $50 \%$ lower odds of developing dementia, and $60 \%$ lower odds of AD development, compared with a sedentary lifestyle in mid life.

These studies indicate that mid-life cardiovascular risk factors influence the risk of developing dementia and AD in later life, which might have important implications for preventive healthcare.

\section{Christine Kyme}

Original articles Kivipelto M et al. (2005) Obesity and vascular risk factors at midlife and the risk of dementia and Alzheimer disease. Arch Neurol 62: 1556-1560

Rovio S et al. (2005) Leisure-time physical activity at midlife and the risk of dementia and Alzheimer's disease. Lancet Neurol 4: 705-711 